\title{
From genes to networks
}

\author{
Sixty years ago, Francis Crick articulated the central dogma of molecular biology to explain the sequential \\ information flow between genes and proteins. Nowadays our understanding of genes and the information they \\ convey is no longer limited to the single-molecule level.
}

T he Cold Spring Harbor Laboratory (CSHL), recently hosted their 11th Plant Genomes meeting while also celebrating the 20th anniversary of their biennial Plant meeting series. This year also marks the 20th anniversary of the National Plant Genome Initiative (NPGI), which has been dedicated to accelerating the basic and translational research of plant genomics. With the support of the NPGI and various global collaborations, Arabidopsis thaliana became the first sequenced plant ${ }^{1}$.

The publication of its genome in 2000 was three years ahead of the completion of the Human Genome Project, marking the dawn of the genome era in plant research. Now there are at least 264 plant species with their reference genomes sequenced and published (http://www.plabipd.de), from superficially simple algae and bryophytes to complex gymnosperms and angiosperms.

Opening the abstract book of the first CSHL Plant Genomes meeting (1997), we can still feel the beat of the Arabidopsis genome project. The sequencing updates of Chromosome 1-5 were made by at least eight international research groups from regions including the United States, the United Kingdom, France and Japan. Despite only partial sequencing data being available, gene annotation and positional cloning were already being pursued to understand the functions of different genes. At this point, the limited resources and tools of functional genomics were serious obstacles for gene studies. It is a favourite joke among early-career scientists today that "if we could take a time machine back to the decade when cloning a gene was sufficient to publish a high-impact article, research life will be much easier". However, the expanding demand for new tools also generated great potential for innovations in methodology and biotechnology.

The first genome-wide transfer DNA mutagenesis of $A$. thaliana in 2003 , led by the Salk Institute, was a major breakthrough in plant functional genomics ${ }^{2}$. This enormous effort directly triggered the blossoming of plant molecular genetics in the past 15 years, and its influence is still strong today. For Arabidopsis researchers, it is now hard to imagine how experiments could be designed and conducted without the seed collection in the Arabidopsis Biological Resource Center (ABRC) or access to the Arabidopsis Information Resource (TAIR).

Rice (Oryza sativa) was the second plant species to have its whole genome sequenced $^{3,4}$. The release of the reference genome of rice and other important crops has provided great opportunities to understand the history of crop domestication and identify landmarks for crop improvement. Combining the knowledge gained from functional genomics and modern plant breeding, scientists have successfully designed and developed new rice varieties with high-yield and superiorquality grains ${ }^{5}$. Similar improvements in other crops are also ongoing.

These achievements and foundations have pushed plant research into the fast lane. The sequencing and assembly of high-quality 'platinum' genomes ${ }^{6-8}$ further empowers plant scientists to pursue the details of genetic regulation and deepen our understanding of plant evolution. For example, in this issue, Wan and colleagues ${ }^{9}$ report a high-quality draft genome of Gnetum montanum, a gymnosperm and the first sequenced species for gnetophytes. They revealed unique genomic features of this species that seem to place it closer to the basal lineage of angiosperms, indicating an early evolutionary path for seed plants.

In addition to these high-quality genomes, revolutionary technologies and tools used for functional genomic analysis are constantly emerging, including new transformation methods, gene-editing tools, yeast two-hybrid systems, mass spectrometry and high-throughput bioinformatics. Combining different methods for functional research is becoming popular, providing a powerful toolkit for digging into the hidden nature of genome sequences. In an article in this issue, $\mathrm{Xu}$ and coworkers ${ }^{10}$ identify a key heterodimer that controls cell reprogramming during Arabidopsis organ regeneration. To test the hypothesis, they performed high-quality analyses using mutant lines from ABRC, transgenic plants, confocal microscopy and approaches to identify both protein-protein interactions and protein-DNA interactions.
Such newly developed technologies liberate researchers from conventional research scenarios and open a new dimension of network thinking. But translating information from genes to proteins is not an easy road. Simply analysing the sequences of genes will never tell the true stories of gene regulation. Using high-throughput chromosome confirmation capture (Hi-C) and chromatin interaction analysis through paired-end tag sequencing (ChIA-PET), Wang et al. ${ }^{11}$ present the $3 \mathrm{D}$ genome architecture of cotton and show how its evolutionary dynamics altered the networks of gene regulation, again in this issue.

If there were a time machine, it would be fascinating to shuttle back to 1997 , to fact-check the memories of the scientists who attended the first CSHL Plant meeting: The Arabidopsis Genome: From Sequence to Function. Whether you regard the intervening 20 years as long or short, they have seen a remarkable accumulation of knowledge and technologies in plant science. The scope of the recent CSHL Plant meeting Plant Genomes \& Biotechnology: From Genes to Networks reflected those changes. With our current ability to do incredible research in an unprecedented landscape, we should never forget the historic path that has led those possibilities, nor fail to keep in mind how important it is to offer shoulders for younger generations to stand on, and see further.

Published online: 31 January 2018 https://doi.org/10.1038/s41477-018-0109-x

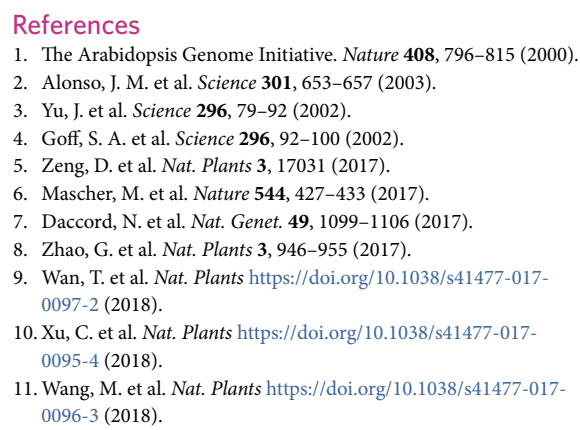

\section{Acknowledgements}

The editorial team acknowledges the support of the Cold Spring Harbor Laboratory, and the Meetings \& Courses Department in particular, for allowing access to the abstract books of the historical meetings. 